\title{
Identification, characterization and expression analysis of transient receptor potential channel genes in the oriental fruit fly, Bactrocera dorsalis
}

Hong-ai Su, Xue Bai, Tian Zeng, Yong-yue Lu* and Yi-xiang Qi

\begin{abstract}
Background: Members of the transient receptor potential (TRP) superfamily are proteins that are critical for insects to detect changes in environmental stimuli and also play key roles in their sensory physiology. Moreover, this family provides potential targets for the design of insecticides. In contrast to a large number of studies conducted on Drosophila melanogaster, molecular studies to characterize TRP channels in agricultural pests are lacking.

Results: In this study, we identified 15 TRP channel genes in the genome of a notorious agricultural pest, the oriental fruit fly (Bactrocera dorsalis). Comparative analysis of the TRP channels (TRPs) in B. dorsalis with those in D. melanogaster, Glossina morsitans, Musca domestica and the closely related Ceratitis capitata, and TRPs from mosquitoes, Hymenoptera, Lepidoptera, Coleoptera and Hemiptera reveals that members of TRPA and TRPP subfamily are most diverse among insects. The results also suggest that Tephritidae family have two TRP-Polycystin 2 members even though most insects either possess just one or none. The highest expression levels of these two genes are in the testes of $B$. dorsalis, implying a role in regulating sperm function. We analyzed the expression profiles of the TRP channels identified in this study at different life stages using quantitative real time PCR. The results of this study demonstrate that all TRP channels are mainly expressed in adults, especially at mature stages. The one exception to this trend is BdTRPM, which is more highly expressed in the eggs of $B$. dorsalis, implying an important role in early development. We also detected the spatial expression of TRP channels in mature adult fruit flies by investigating expression levels within various tissues including those involved in sensory function, such as antennae, compound eyes, mouthparts, legs, and wings, as well as tissues critical for homeostasis and physiology (i.e., Malpighian tubules, the brain and gut as well as fat bodies, ovaries, and testes).
\end{abstract}

Conclusion: The results of this study establish a solid foundation for future functional characterization of $B$. dorsalis TRP channels as well as those of other insects and will help future insecticide design targeting these channels.

Keywords: Bactrocera dorsalis, Transient receptor potential, Cationic channels, Splice forms, Expression analysis

\section{Background}

Transient receptor potential (TRP) superfamily proteins are six transmembrane domain cationic channels with some calcium permeability, implicated in many cellular functions [1]. This superfamily, whose members are found in all animals, can be activated by a variety of mechanisms and play critical roles in sensory physiology

\footnotetext{
* Correspondence: luyongyue@scau.edu.cn; qiyixiang@scau.edu.cn Department of Entomology, College of Agriculture, South China Agricultural University, Wushan Road 483, Tianhe District, Guangzhou 510642, China
}

including vision, hearing, taste, touch, gravity, olfaction, humidity, thermo- and osmosensation $[1,2]$. In insects, these channels have a profound impact on behaviors and physiological functions [2-5].

The first TRP channel to be identified was Drosophila TRP; this family member was initially identified and characterized in phototransduction about three decades ago [6]. Numerous TRP-like channels have subsequently been identified in eukaryotes; these are arranged into seven subfamilies based on their primary amino acid

(c) The Author(s). 2018 Open Access This article is distributed under the terms of the Creative Commons Attribution 4.0 International License (http://creativecommons.org/licenses/by/4.0/), which permits unrestricted use, distribution, and reproduction in any medium, provided you give appropriate credit to the original author(s) and the source, provide a link to the Creative Commons license, and indicate if changes were made. The Creative Commons Public Domain Dedication waiver (http://creativecommons.org/publicdomain/zero/1.0/) applies to the data made available in this article, unless otherwise stated. 
sequence homology, TRP-Canonical (TRPC), TRP-Ankyrin (TRPA), TRP-No mechanoreceptor potential C (TRPN), TRP-Vanilloid (TRPV), TRP-Melastatin (TRPM), TRPMucolipin (TRPML), and TRP-Polycystin (TRPP) [1, 2]. These seven subfamilies are themselves broadly divided into two groups with TRPC, TRPA, TRPN, TRPV, and TRPM classified as group 1 TRPs because they share the most sequence similarity with the founding member of this superfamily, Drosophila TRP. In contrast, group 2 TRPs comprise the TRPP and TRPML subfamilies that are distantly related to their group 1 counterparts [1]. Sixteen TRP members have been identified and characterized in Drosophila. The TRPC subfamily comprises TRP, TRP-Like (TRPL), and TRPgamma (TRPY). The TRPA subfamily includes TRPA1, Painless (Pain), Pyrexia (Pyx), and Waterwitch (wtrw), while the TRPN subfamily has just a single member that is not found in mammals, No mechano-receptor potential C (NompC). The TRPV subfamily includes inactive (Iav) and Nanchung (Nan), while TRPM is the only member of the subfamily bearing this name. The TRPP subfamily has polycystin-2 (Pkd2) and brivido (Brv). In contrast to most TRP members which have 6 transmembrane domains, Brv proteins contain between 8 and 10 transmembrane segments and are not known to form ion channels independently previously [7]. But recently, the Drosophlia Brv1 is proved to forms a mechanosensitive cation channel and is essential for gentle-touch sensation [8]. Finally, TRPML subfamilies only contain TRP Mucolipin $[1,2]$. Previous research has demonstrated the presence of a diverse range of TRP superfamily members amongst insect species [9]; if the Brv genes are not counted, most insects possess between 13 and 14 TRP components, approximately half the number in mammals [10].

The oriental fruit fly, Bactrocera dorsalis (Hendel) (Diptera: Tephritidae), is a polyphagous pest. This species is recognized as one of the most destructive fruit industry pests because of its wide distribution, rapid invasiveness, and the high level of damage it causes [11, 12]. As the long-term and excessive use of chemical insecticides to mitigate the problems caused by this species have led to serious resistance issues $[13,14]$, it is now urgent to develop alternative targets. In this context, TRP channels have become key insecticide targets because of their critical physiological and cellular functions. Previous researches have shown that both pymetrozine and afidopyropen act by modulating TRPV channels $[15,16]$, and that TRPA1 in the mosquito vector for malaria, Anopheles gambiae, is potently and directly activated by citronellal [17]. Thus, TRPs have become potential targets for insecticide development as well as for improved repellents to control insect-borne diseases $[18,19]$. Investigating the molecular characteristics of TRPs in $B$. dorsalis will enable a better understanding of this system in a key agricultural pest and will provide a firm foundation for future insecticide design targeting these channels.

In this study, we identified 15 TRP members in B. dorsalis genome and transcriptome data deposited in the GenBank database and examined the expression patterns of these TRPs at different developmental stages and in various $B$. dorsalis tissues.

\section{Results \\ Identification, sequence analysis, and splice variants of TRP channels in $B$. dorsalis}

We identified 15 TRP channel genes in $B$. dorsalis that share homology with known Drosophila TRP channel sequences. Phylogenetic analysis reveals that these channels include three TRPC, four TRPA, one TRPN, two TRPV, one TRPM, three TRPP, and one TRPML subfamily members, respectively (Fig. 1 and Table 1). Sequence analysis revealed the presence of six transmembrane domains in all $B$. dorsalis TRPs with the exception of BdorBrv, which has eight transmembrane segments (Table 1). Most of group-1 TRPs possess multiple $\mathrm{N}$-terminal ankyrin repeats domain (Table 1); BLASTP analyses of protein sequence alignments show that all $B$. dorsalis TRPs have a high level of sequence identity (above $50 \%$ ) versus those in D. melanogaster, with the exception of the TRPP subfamily members (Table 1). Data show that while Drosophila has just one $P k d 2$ gene, B. dorsalis has two; BdorPkd2-1 and BdorPkd2-2. The Mediterranean fruit fly, Ceratitis capitata also possess two $P k d 2$ genes, while other insects we investigated just have one or none (Table 2). We validated the ORF sequence of $B d o r P k d 2-1$ and $B d o r P k d 2-2$ via RT-PCR; compared with DmelPkd2, both these genes have a much shorter N-terminal (Fig. 2) and contain a large loop that separates the first two transmembrane domains (Fig. 2), a characteristic feature of group-2 TRPs [1]. No ankyrin repeats were detected in either BdorPkd2-1 or BdorPkd2-2 (Fig. 2 and Table 1). We only identified one Brv gene in B. dorsalis and other Diptera insects we investigated, with the exception of $D$. melanogaster, which has three (Table 2). Although we also identified one Brv gene in Tribolium castaneum (XP_015838037.1), it doesn't cluster with other Brv proteins (Fig. 1). The reason May be that the sequence of the transmembrane segments of this protein is incomplete. To confirm whether Brv proteins are specific to fly species, the genomes of more insect species need to be investigated and the complete sequence of the identified Brv gene in T. castaneum need to be obtained and characterrized. The numbers of TRP superfamily members among different insect species are varied, even in the same order, such as Diptera (Table 2).

As we identified splice variants for BdorTRPL, we performed RT-PCR on compound eyes using specific 


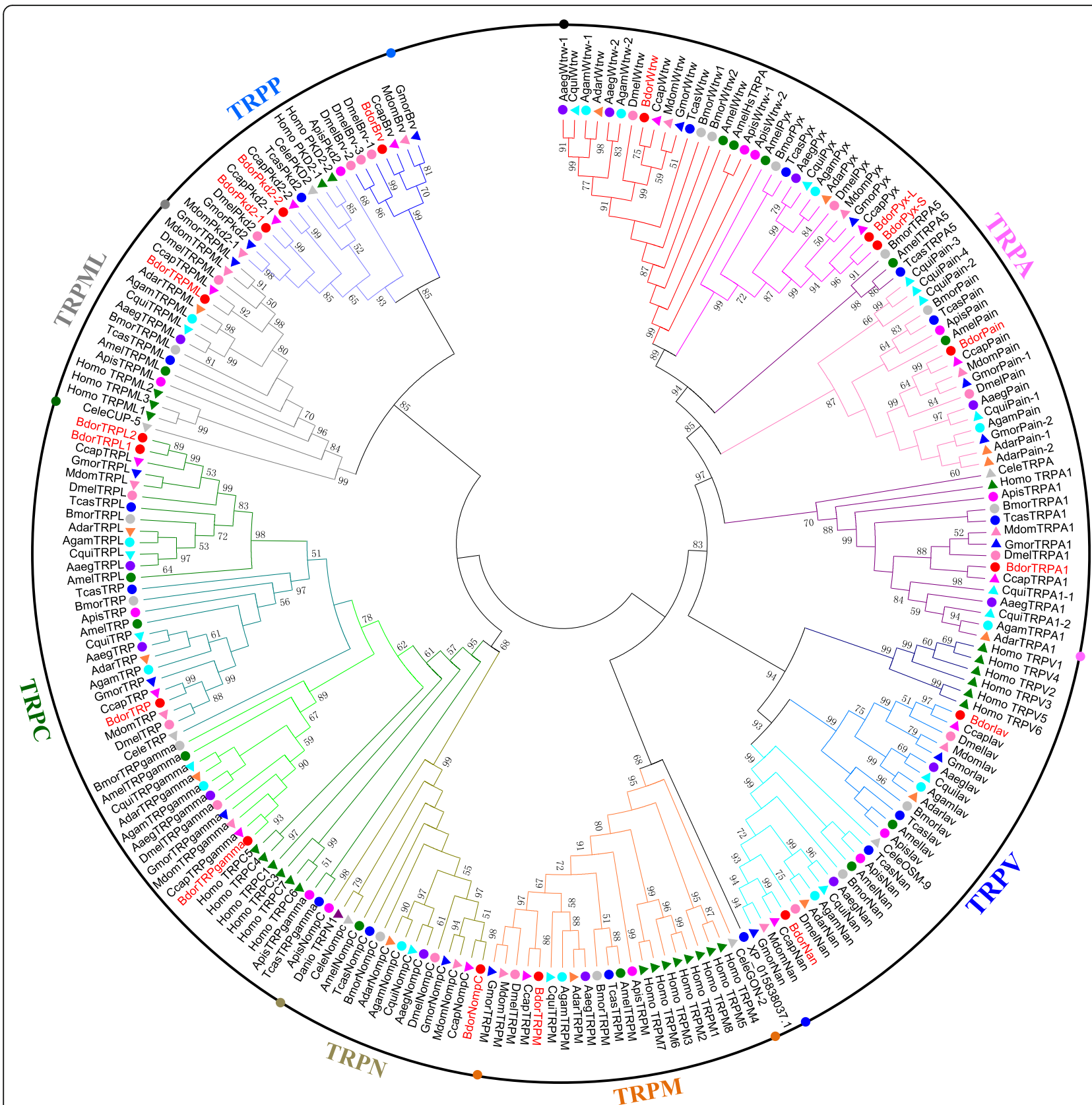

Fig. 1 Phylogenetic analysis of TRP channels in B. dorsalis and other insects. The tree was constructed using the software MEGA 5.05 with 1000 bootstrap replicates based on the Maximum Likelihood method. The numbers on branch nodes denote levels of bootstrap support. Species abbreviations are Aaeg, Aedes aegypti, Adar, Anopheles darlingi, Agam, Anopheles gambiae, Amel, Apis mellifera, Apis, Acyrthosiphon pisum, Bdor, Bactrocera dorsalis, Bmor, Bombyx mori, Ccap, Ceratitis capitata, Cqui, Culex quinquefasciatus, Cele, Caenorhabditis elegans, Dmel, Drosophila melanogaster, Danio, Danio rerio, Gmor, Glossina morsitans, Homo, Homo sapiens, Mdom, Musca domestica, Tcas, Tribolium castaneum

primers and sequenced the products to confirm their form. The results of this step show that at least two splice forms of BdorTRPL are present; transcripts of BdorTRPL1 are more abundant than those of BdorTRPL2 in the compound eyes of $B$. dorsalis (Fig. 3a). We therefore further analyzed the genome and transcriptome data deposited in the NCBI database to investigate the positions of splicing sites that would be expected to explain the generation of identified forms. The splice forms were generated by two mutually exclusive exons; the second and third (Fig. $3 \mathrm{~b})$; thus, the ORF of BdorTRPL1 is 3744 base pairs (bp) and codes for a protein that comprises 1247 amino-acid residues. Data show that BdorTRPL2 contains an ORF comprising 3755 bp that codes for a protein consisting of 1250 amino-acid residues. 
Table 1 TRP channels identified from $B$. dorsalis

\begin{tabular}{|c|c|c|c|c|c|c|c|c|}
\hline Subfamily & $\begin{array}{l}\text { Gene } \\
\text { name }\end{array}$ & $\begin{array}{l}\text { Genomic } \\
\text { Sequence ID }\end{array}$ & $\begin{array}{l}\text { NCBI } \\
\text { accession no. } \\
\text { (Transcripts) }\end{array}$ & $\begin{array}{l}\text { Length } \\
\text { (amino } \\
\text { acids) }\end{array}$ & $\begin{array}{l}\text { Protein region } \\
\text { identified (TM) }\end{array}$ & $\begin{array}{l}\text { Number of } \\
\text { ankyrin } \\
\text { repeats }\end{array}$ & $\begin{array}{l}\text { CG no. of the } \\
\text { D. melanogaster } \\
\text { orthologue }\end{array}$ & $\begin{array}{l}\text { Sequence identity } \\
\text { between } \\
\text { B. dorsalis and } D \text {. } \\
\text { melanogaster }\end{array}$ \\
\hline \multicolumn{9}{|c|}{ Group-1 TRPs } \\
\hline \multirow[t]{3}{*}{ TRPC } & BdorTRP & NW_011876214.1 & XM_011199037 & 1255 & TM1-6 & 4 & CG7875 & $75 \%$ \\
\hline & BdorTRPL & NW_011876127.1 & XM_019992825 & 1250 & TM1-6 & 4 & CG18345 & $70 \%$ \\
\hline & BdorTRP $\gamma$ & NW_011876374.1 & XM_019991203 & 1148 & TM1-6 & 4 & CG5996 & $86 \%$ \\
\hline \multirow[t]{4}{*}{ TRPA } & BdTRPA1 & NW_011876386.1 & XM_011210731 & 1244 & TM1-6 & 13 & CG5751 & $79 \%$ \\
\hline & BdorPain & NW_011876390.1 & XM_011211573 & 930 & TM1-6 & 9 & CG15860 & $52 \%$ \\
\hline & BdorPyx & NW_011876344.1 & XM_011205344 & 975 & TM1-6 & 9 & CG17142 & $73 \%$ \\
\hline & BdorWtrw & NW_011876251.1 & XM_011200137 & 981 & TM1-6 & 9 & CG31284 & $89 \%$ \\
\hline TRPN & BdorNompC & NW_011876379.1 & XM_019991381 & 1705 & TM1-6 & 29 & CG11020 & $88 \%$ \\
\hline \multirow[t]{2}{*}{ TRPV } & Bdorlav & NW_011876375.1 & XM_011208858 & 897 & TM4-6 & 5 & CG4536 & $85 \%$ \\
\hline & BdorNan & NW_011876301.1 & XM_019989557 & 832 & TM1-6 & 5 & CG5842 & $85 \%$ \\
\hline TRPM & BdorTRPM & NW_011876216.1 & XM_019988876 & 2095 & TM1-6 & 0 & CG44240 & $77 \%$ \\
\hline \multicolumn{9}{|c|}{ Group-2 TRPs } \\
\hline \multirow[t]{3}{*}{ TRPP } & BdorPkd2-1 & NW_011876199.1 & XM_011216470 & 662 & TM1-6 & 0 & CG6504 & $39 \%$ \\
\hline & BdorPkd2-2 & NW 011876391.1 & XM 011211817 & 639 & TM1-6 & 0 & CG6504 & $37 \%$ \\
\hline & BdorBrv & NW_011876251.1 & XM_011200123 & 711 & TM1-8 & 0 & CG13762 & $38 \%$ \\
\hline TRPML & BdorTRPML & NW_011876336.1 & XM_011204634 & 695 & TM1-6 & 0 & CG8743 & $72 \%$ \\
\hline
\end{tabular}

Sequence differences between BdorTRPL1 and BdorTRPL2 include between 18 and $21 \mathrm{~N}$-terminal amino-acid residues (Fig. 3c). The amino acid sequence of BdorTRPL comprises six transmembrane domains (i.e., TM1 to TM6) and four ankyrin repeats. The TRP domain, which follows the sixth transmembrane segment, was detected in BdorTRPL. TRP box 1 and TRP box 2 are the most conserved portions of this domain (Fig. 3c) [1].
We also detected two splice variants for BdorPyx by performing RT-PCR on abdomen samples. Data reveal a higher long form (BdorPyx-L) expression level compared to the short form (BdorPyx-S) (Fig. 4a); the ORF of $B d o r P y x-L$ comprises 2928 bp and codes for a protein consisting of 975 amino-acid residues, while that of $B d o r P y x-S$ is 2553 bp in length and codes for a protein of 850 amino-acid residues. This splicing is generated by

Table 2 Number of TRP subfamily members in the genome of $B$. dorsalis and several other Diptera insects

Number of TRP subfamily members in the genome of $B$. dorsalis and several other Diptera insects

\begin{tabular}{|c|c|c|c|c|c|c|c|c|c|c|c|c|c|c|c|c|c|}
\hline \multirow{3}{*}{ Species name } & \multicolumn{16}{|c|}{ Channel type } & \multirow{3}{*}{ Total } \\
\hline & TRPC & & & TRPA & & & & & & TRPN & TRP & & TRPM & TRPP & & TRPML & \\
\hline & TRP & TRPL & TRP $\gamma$ & TRPA1 & TRPA5 & HsTRPA & Pain & Pyx & Wtrw & NompC & Iav & Nan & TRPM & Brv & Pkd2 & TRPML & \\
\hline
\end{tabular}

Diptera

Fly

\begin{tabular}{|c|c|c|c|c|c|c|c|c|c|c|c|c|c|c|c|c|}
\hline \multirow[t]{2}{*}{ Drosophila melanogaster } & 1 & 1 & 1 & 1 & $\mathbf{0}$ & $\mathbf{0}$ & 1 & 1 & 1 & 1 & 1 & 1 & 1 & 3 & 1 & 1 \\
\hline & 1 & 1 & 1 & 1 & 0 & $\mathbf{0}$ & 2 & 1 & 1 & 1 & 1 & 1 & 1 & $\mathbf{1}$ & 1 & 1 \\
\hline Musca domestica & 1 & 1 & 1 & 1 & $\mathbf{0}$ & $\mathbf{0}$ & 1 & $\mathbf{1}$ & 1 & 1 & 1 & 1 & 1 & 1 & 1 & 1 \\
\hline Bactrocera dorsalis & 1 & 1 & 1 & 1 & 0 & $\mathbf{0}$ & 1 & 1 & 1 & 1 & 1 & 1 & 1 & 1 & 2 & 1 \\
\hline Ceratitis capitata & 1 & 1 & 1 & 1 & $\mathbf{0}$ & $\mathbf{0}$ & 1 & 1 & 1 & 1 & 1 & 1 & 1 & 1 & 2 & 1 \\
\hline
\end{tabular}

Mosquito

Aedes aegypti

Anopheles gambiae

Anopheles darlingi

Culex quinquefasciatus

Hymenoptera

Apis mellifera

Lepidoptera

Bombyx mori

Coleoptera

$\begin{array}{lllll}1 & 1 & 1 & 1 & 0\end{array}$

$\begin{array}{lllll}1 & 1 & 1 & 1 & 0 \\ 1 & 1 & 1 & 1 & 0 \\ 1 & 1 & 1 & 1 & 0 \\ 1 & 1 & 1 & 2 & 0\end{array}$

$\begin{array}{lllll}\mathbf{0} & \mathbf{1} & \mathbf{1} & \mathbf{2} & \mathbf{1} \\ \mathbf{0} & \mathbf{1} & \mathbf{1} & \mathbf{2} & 1 \\ \mathbf{0} & 2 & 1 & 1 & 1 \\ \mathbf{0} & 4 & 1 & 1 & 1\end{array}$

$\begin{array}{ll}1 & 1 \\ 1 & 1 \\ 1 & 1 \\ 1 & 1\end{array}$

15

Hemiptera

Acyrthosiphon pisum 


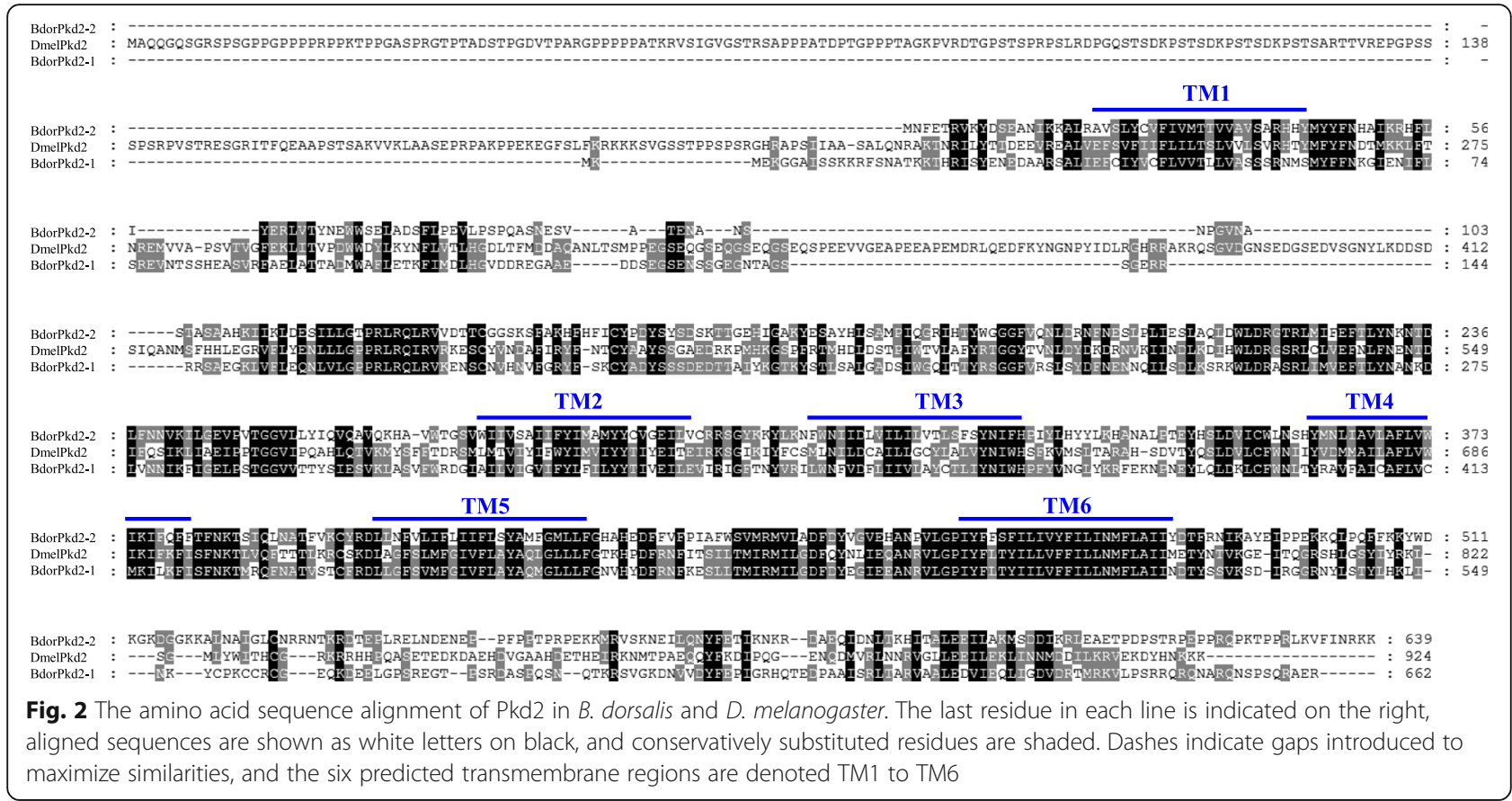

the excision of a $375 \mathrm{bp}$ fragment within the sixth exon (Fig. 4b). Thus, compared to the six transmembrane domains seen in BdorPyx-L, this excision is potentially responsible for the generation of the truncated polypeptide in BdorPyx-S that just possesses four (Fig. 4c).

\section{TRP channel transcript levels in different $B$. dorsalis developmental stages}

We used qRT-PCR to investigate the levels of temporal expression in B. dorsalis TRPs. Multiple stages were tested in this study including eggs, larvae, pupae, immature (one day old), and mature (13 days old) adults. Results reveal that BdorTRP is expressed to a relatively high level in adult stages as well as in seven day old pupae (Fig. 5a). Although adults possess abundant transcripts of both the two BdorTRPL splice forms (Figs. 5b and c), BdorTRPL1 is expressed in adults while BdorTRPL2 is also expressed in larvae and pupae. We were barely able to detect BdorTRPy in eggs even though this form was commonly present in all other tested stages (Fig. 5d). Immature adults also express abundant BdorTRPA1, and transcripts of this channel were more common in mature females than males (Fig. 5e). BdorPain is more highly expressed in adults than in other stages (Fig. 5f). Results show that the long and short forms of $B$ dorPyx were expressed differently depending on $B$. dorsalis life stage; both larval and pupal stages mainly express the short form transcripts while adults mainly express the long form (Fig. 5g). Indeed, similar to BdorTRP, abundant BdorWtrw transcripts were also detected in seven day old pupae and adults (Fig. 5h), while BdorNompC, BdorIav,
BdorNan, BdorPkd2-1, and BdorPkd2-2 were all more highly expressed in mature males compared to all other stages (Figs. 5i, j, k, n and o). BdorBrv is widely expressed across all the tested stages except eggs (Fig. 5m). Data show that just BdorTRPM was highly expressed in eggs among tested TRPs (Fig. 51), while mature females expressed the highest number of BdorTRPML transcripts (Fig. 5o). All the TRPs we tested are mainly expressed in adults, with the exception of BdorTRPM which is expressed to a greater extent in eggs than in other stages.

\section{Tissue distribution of TRP channels in B. dorsalis}

Insect external structures, including antennae, compound eyes, mouthparts, legs, and wings, are all important for sensing environmental stimuli. The internal tissues such as Malpighian tubules, brain, gut and fat bodies as well as ovaries and testes are critical for insect homeostasis. To investigate the possible roles of the identified TRPs in $B$. dorsalis, we therefore measured TRP expression levels in different tissues of mature adults. Results show that transcripts of BdorTRP, BdorTRPL1, and BdorTRPL2 are much higher in compound eyes and brains than in other tissues (Figs. 6a-b). In compound eyes and mouthparts, mRNA level of BdorTRPL1 is higher than that of BdorTRPL2 (Figs. 6b). While higher transcripts of BdorTRPL2 were detected in wing, fat body, gut, Malpighian tubules, ovaries and testes than BdorTRPL1 in these tissues (Figs. 6b). BdorTRPy is mainly expressed in wings, legs and brains (Fig. 6c). In contrast, hardly any transcripts of BdorTRPA1 were found in wings and legs, but are abundant in antennae, mouthparts, brains and gut (Fig. 6d). Data 


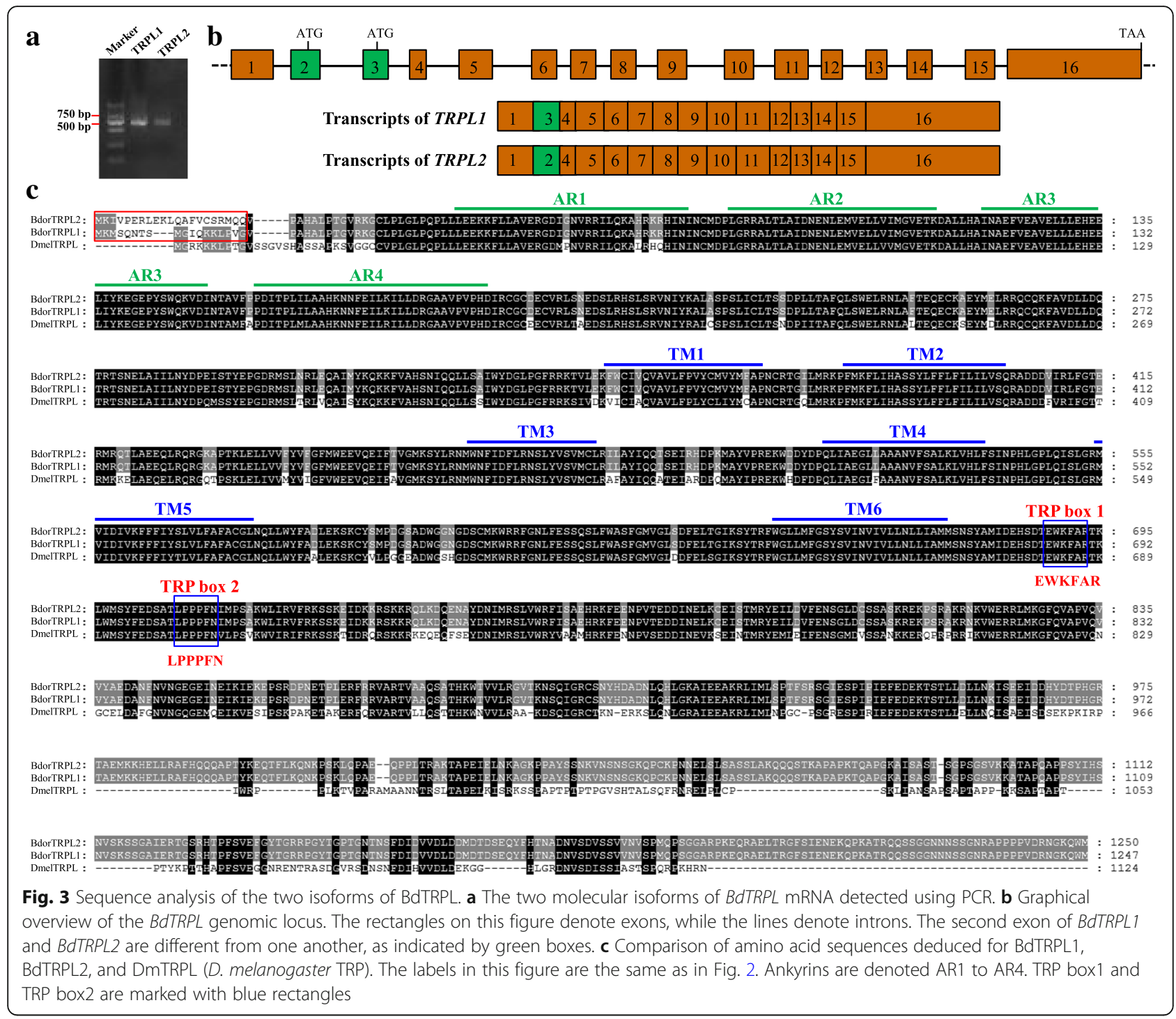

also show that BdorPain is expressed to a high level in legs (Fig. 6e), while BdorPyx is mainly expressed in the antennae of $B$. dorsalis. It is also noteworthy that primer-amplified BdorPyx comprises two isoforms which coexpressed in this analysis in a ratio strongly favoring the longer variety in compound eyes, legs, brains, fat body, ovaries and testes (Fig. 6f). High messenger RNA (mRNA) levels of BdorWtrw, BdorIav, and BdorNan were also found in legs and wings (Figs. $6 \mathrm{~g}$, $\mathrm{i}$ and $\mathrm{j}$ ). The highest expression level of both BdWtrw and BdNompC were detected in brains (Figs. $6 \mathrm{~g}$ and $\mathrm{h}$ ). Abundant transcripts of BdorTRPM and BdorTRPML were detected in Malpighian tubules (Figs. 6k and p), while a high BdorTRPM and BdorTRPML mRNA level was also found in ovaries and gut respectively (Fig. $6 \mathrm{k}$ and p). The highest expression levels of BdorBrv, BdorPkd2-1 and BdorPkd2-2 were observed in testes in all cases (Fig. 6m-o). Apart from testes, BdorBrv is also highly expressed in fat bodies and legs (Fig. $6 \mathrm{~m}$ ).

\section{Discussion}

We identified $15 \mathrm{~B}$. dorsalis TRPs in this study that can be divided into seven subfamilies on the basis of their structure and phylogenetic analyses. Our data show that $B$. dorsalis possesses three TRPP members including BdorBrv, BdorPkd2-1 and BdorPkd2-2. The numbers of TRPP channels are known to vary amongst insect species; while most contain just one $P k d 2$ gene (e.g., Drosophila), this channel is entirely absent in some lepidopterans and hymenopterans (e.g., Bombyx and Apis) (Table 2) [10]. D. melanogaster have three Brv genes, but we detected only one Brv gene in $B$. dorsalis. The rest insects we investigated also have one Brv or none. It is thought that TRPP comprises the most ancient TRP subfamily because members of this group are known from taxa spanning yeast to mammals $[20,21]$. However, the sequence identities of TRPP members between $B$. dorsalis and $D$. melanogaster are relatively low; previous studies have shown that the 


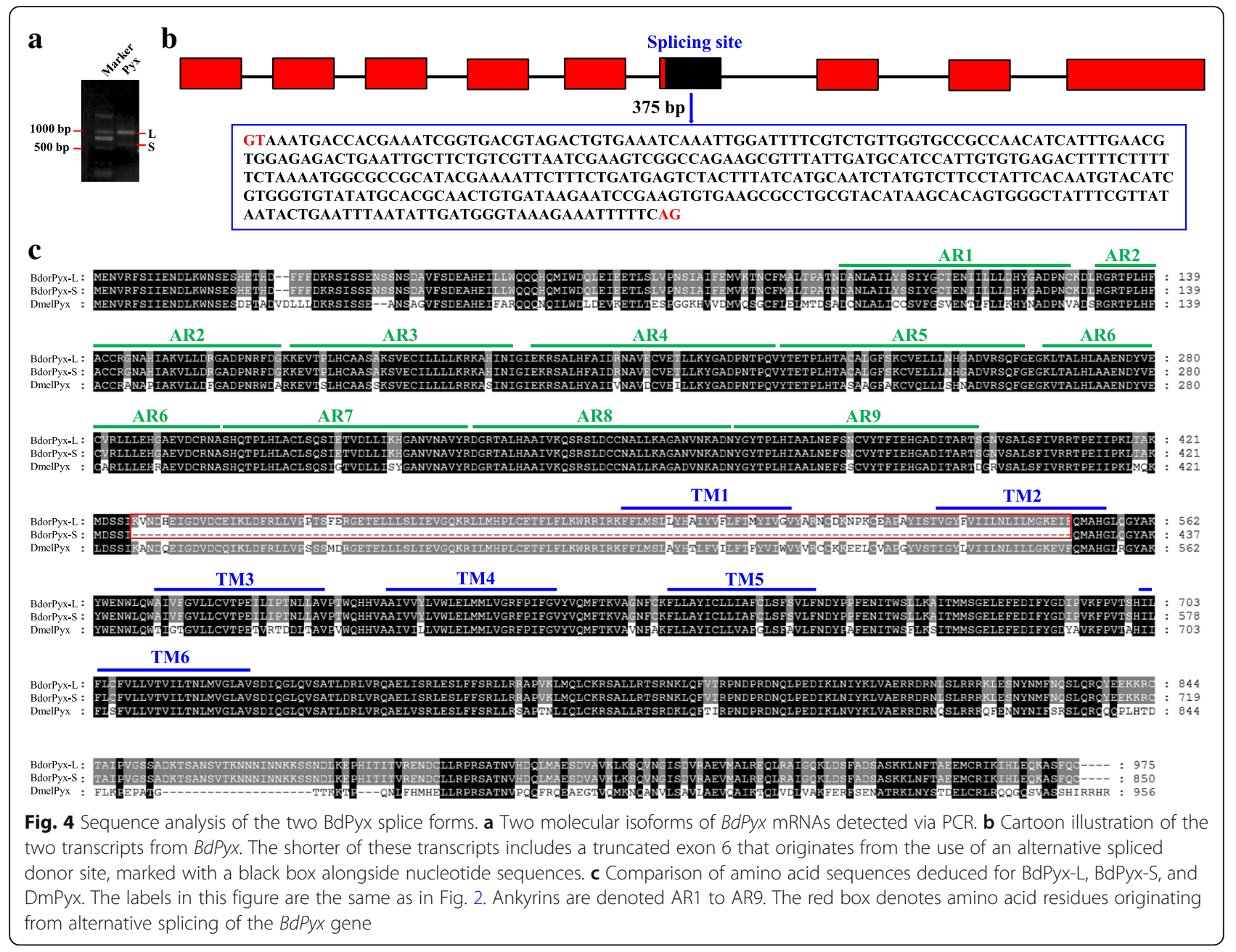

evolutionary rate of TRPP channels has been accelerated relative to other TRPs and members of this subfamily might perform different physiological functions in distinct insect species [10,22]. Although $B$. dorsalis have the same number of TRPA genes with D. melanogaster, members of this subfamily are most diverse among arthropod species, particularly in insects [9]. In this study, more TRPA gens were detected in mosquitos than in flies. The TRPA1 gene was lost in Hymenoptera, but insects belonging to this order have Hymenoptera- specific TRPA (HsTRPA) probably compensates for the lack of TRPA1 [10, 23]. TRPA subfamily members are involved in sensing compounds, temperature and humidity. The more complicated lifestyle an insect live, the more TRPA members they may have. Due to the expansion of Pain and TRPA5 channels, the social insect $S$. invicta have 27 TRP genes, much more than most insects [9]. The amplification and reduction of TRPs in different insects indicate that the evolution of this superfamily is related to specific habitats and life histories of individual species. Future studies are needed to investigate the physiological significance of the expanded TRPs.
All of the TRPs detected in this study are highly expressed in adults (especially mature individuals), with the exception of BdTRPM. We know that these channels are critical to just about every sensory modality in insects and so impact behaviors as diverse as phototaxis, thermotaxis, gravitaxis, the avoidance of noxious tastants and smells, and proprioception [2]. The biology of $B$. dorsalis adults and the environmental conditions within which they live are more diverse than their other life stages; indeed, multiple behaviors are only seen in adult flies, including courtship, mating, flight, and egg-laying. These observations are important because while most TRPs could barely be detected in eggs, the highest TRPM expression level was found during this life stage; this channel may be required for early $B$. dorsalis development as it is critical for $\mathrm{Mg}^{2+}$ and $\mathrm{Zn}^{2+}$ homeostasis $[24,25]$ and members of this subfamily are necessary for initial embryonic development in mice [26].

The data presented here show that $B d T R P$ and the two splice forms of $B d T R P L$ have high levels of expression in compound eyes and the brain, suggesting their 


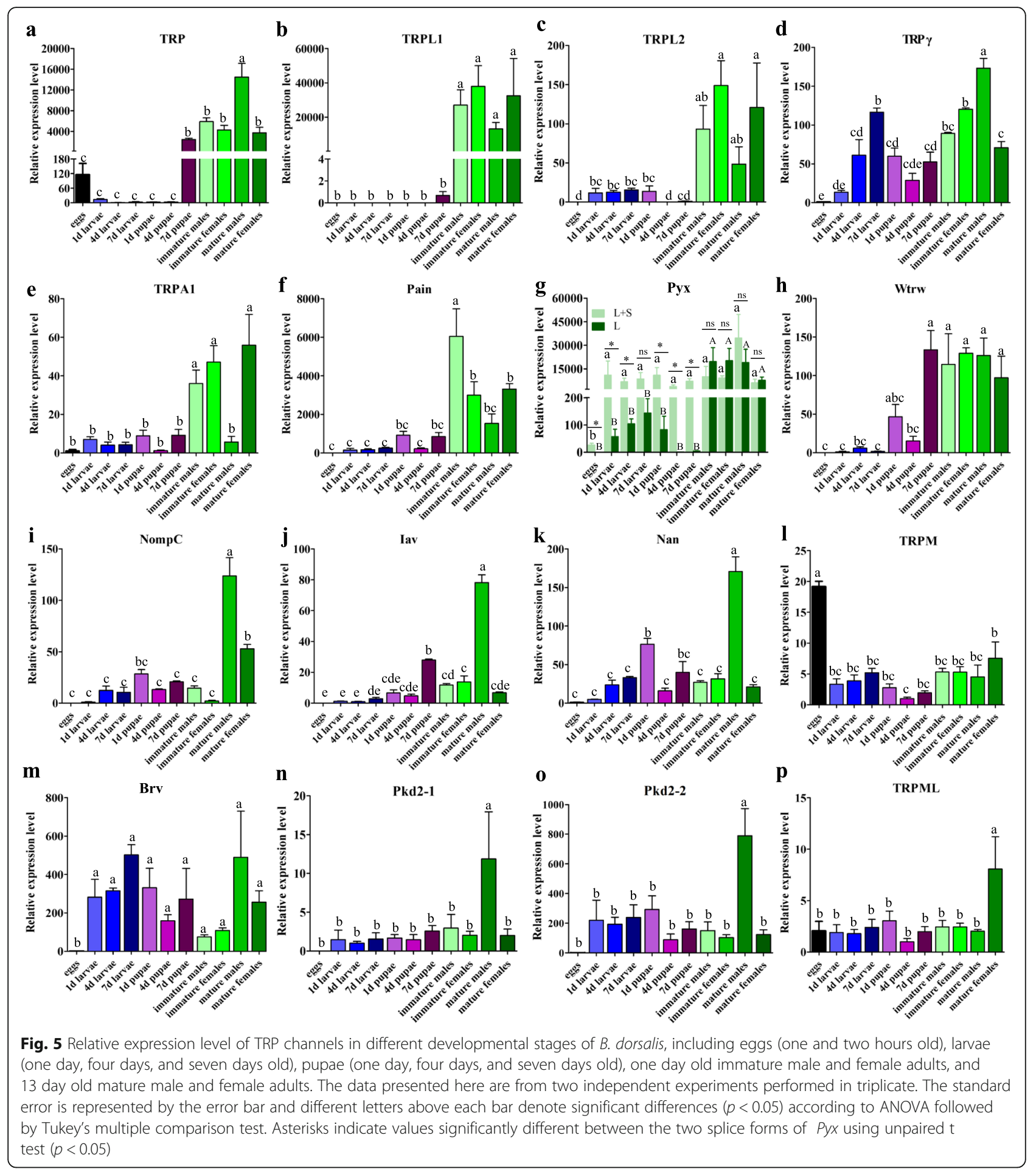

roles in light sensation. Drosophila TRP is expressed predominantly within rhabdomeric membranes of photoreceptor cells and is required for light responses [6]. At the same time, TRPL also participates in phototransduction and is responsible for remaining light responses in the $\operatorname{trp}$ mutant [27]. Interestingly, we detected abundant transcripts of TRPL1 in the mouthparts, suggesting that this channel might modulate $B$. dorsalis feeding. The two spice forms of BdorTRPL are differentially expressed. BdorTRPL2 may regulate the function of fat bodies, gut, Malpighian tubules. The TRPy channel is also highly enriched in photoreceptor cells in Drosophila and is therefore thought to be essential to the phototransduction process [28]. Although no TRP $y$ expression was observed in previous work in the 


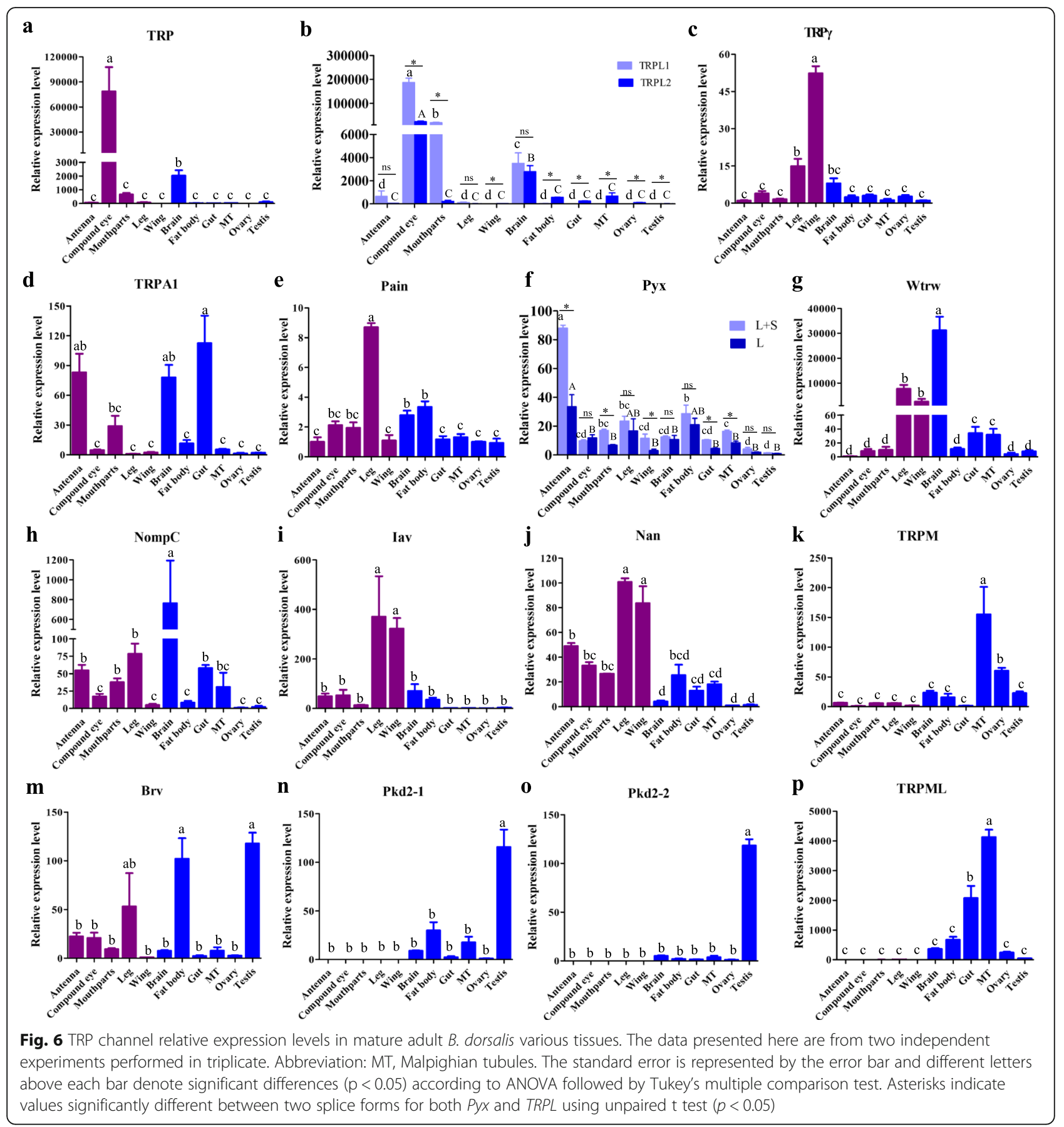

eyes of Spodoptera littoralis [5], we were able to detect low expression in $B$. dorsalis compound eyes. It is also noteworthy that TRPy mRNA is abundant in B. dorsalis wings and legs; proprioceptive neurons are distributed in the joints of appendages in fruit flies, including in the legs and wings [29], where TRPY is expressed in proprioceptive organs and contributes to fine motor control [30]. Moderate TRPy transcripts were detected in the brain of $B$. dorsalis, while in S. littoralis and Periplaneta Americana, TRPy is highly expressed in the brain and the central nervous system [5, 31]. TRPA1 is involved in numerous sensory processes, including temperature sensation and the avoidance of noxious heat, aversive odorants, tastants, non-volatile irritants, bright lights, and mechanical stimuli $[2,32]$. The presence of sensory organs for smell, hearing, and gravity in insect antennae [2] may therefore explain the relatively high $B$. dorsalis TRPA1 expression level in these tissues. Dominant expression of TRPA1 homologs in antennae has been reported previously [33, 34], and we also show robust expression of TRPA1 in $B$. dorsalis gut samples. 
TRPA1 channel may play a critical role in $B$. dorsalis gut immune responses because it promotes the expulsion of bacteria from the gut via a uracil/Duox pathway and is also required for intestinal stem cell proliferation in response to oxidative stress [35, 36]. Similar to TRPA1, Pain is widely distributed in $B$. dorsalis and is also important for the sensation of gravity and the avoidance of noxious heat, mechanical stimulation, and dry environments [2]. We therefore hypothesize an extended role for BdorPain via its involvement in signaling pathways that control various physiological processes. Mechanosensation allows animals to respond to soft touches, noxious sensations, sound, and gravity, and may also contribute to hygrosensation, while Pyx is involved in gravity sensation [2], Wtrw is necessary to detect dry air [37], and NompC is important for light touches, locomotion, and hearing [2, 29]. Organs used for mechanosensation are distributed throughout insect legs and wings, coincident with our findings that relatively high expression levels of Pyx, Wtrw, and NompC also occur in the legs of $B$. dorsalis. Pyx also regulates resistance to noxious heat [38]; the highest level of expression for this channel was detected in B. dorsalis antennae. Similarly, the TRPV channels Iav and Nan are expressed in Johnston's organ and play key roles in gravity and sound sensation [2]; we detected moderate expression levels of both these channels in $B$. dorsalis antennae alongside high levels in legs and wings. The distribution of Iav and Nan in internal tissues is also indicative of their potential roles in regulating $B$. dorsalis physiological processes; as noted, TRPM is essential for $\mathrm{Mg}^{2+}$ and $\mathrm{Zn}^{2+}$ homeostasis and D. melanogaster knocked out this channel have shortened Malpighian tubules [25]. This gene is also essential for life in these flies as mutations result in the death of pupae [25]. Indeed, TRPM is expressed at a high level in $B$. dorsalis Malpighian tubules as well as in several other external structures, suggestive of a role in $\mathrm{Mg}^{2+}$ and $\mathrm{Zn}^{2+}$ homeostasis and the sensation of environmental stimuli. Studies revealed that Drosophila TRPM functions in noxious cold sensation and gentle touch mechanosensation [22]. Given the highly expression level of Brv in testes, fat bodies and legs, this gene might regulate $B$. dorsalis sperm function, immune response, taste and mechanosensation. In Drosophila, Brv genes have been implicated in cool sensation in adults and Brv-1 is required for gentle touch $[8,39]$. Previous evidence showed that the antennae contribute to cold sensation [2]. Moderate Brv expression level was detected in $B$. dorsalis antennae suggesting this protein may also regulate cool sensation in this species. A single Pkd2 is present in Drosophila which localizes to the flagellated sperm tail where it is required for storage [40, 41]. Although TRPP is absent from the genome of several insects such as Bombyx mori, Anopheles darling and Culex quinquefasciatus (Table 2) [9], B. dorsalis has two of these channels both expressed to a high level in testes. We hypothesize that these two Pkd2 members perform different roles in sperm function. TRPML participates in locomotion, autophagy, and the clearance of apoptotic cells [2]. This channel is highly expressed in $B$. dorsalis Malpighian tubules and gut, therefore it might regulate the functions of these two tissues in the oriental fruit fly.

\section{Conclusion}

The number and function of TRPs varies amongst insect species [9]. In this study, we have investigated the members of TRPs in the genome of the notorious agricultural pest, $B$. dorsalis and several other Diptera species. We also detected the expression patterns of TRPs in B. dorsalis. The results of this research expand our knowledge of these critical sensory channels and provide key additional information that will prove valuable to future molecular-level functional studies. This study also lays the foundations for the future development of novel strategies to safely and efficiently control this key insect pest species.

\section{Methods \\ Insects}

Individual $B$. dorsalis flies were reared at $27{ }^{\circ} \mathrm{C} \pm 1{ }^{\circ} \mathrm{C}$ and at $75 \% \pm 1 \%$ relative humidity; all individuals were subjected to a photoperiod cycle comprising $14 \mathrm{~h}$ of light and ten hours of dark. Hatched larvae were maintained on an artificial diet [42]. Larvae were then transferred into small plastic boxes containing sand before pupation and pupae were kept at $27{ }^{\circ} \mathrm{C} \pm 1{ }^{\circ} \mathrm{C}$ until adults emerged. Adult flies were fed another artificial diet comprising yeast extract and dry sugar mixed at a 1:1 ratio $(w / w)$ and housed in wooden cages measuring $35 \mathrm{~cm}$ by $35 \mathrm{~cm}$ by $35 \mathrm{~cm}$ [43].

\section{Identification of TRP channels}

To search exhaustively all TRP genes in each species, we screened several types of database including assembled genomes, reference sequence (RefSeq) database from National Center for Biotechnology Information (NCBI) (https://www.ncbi.nlm.nih.gov/refseq/) and transcriptomic data acquired from NCBI Sequence Read Archive (SRA) Databases (https://www.ncbi.nlm.nih.gov/genbank/ tsa/). The $B$. dorsalis genome has been available from the United States Department of Agriculture National Agricultural Library Database (https://i5k.nal.usda.gov/Bactrocera_dorsalis). The genomes of Glossina morsitans, Aedes aegypti, Anopheles darling, Anopheles gambiae and Culex quinquefasciatus has been available from vectorBase (https://www.vectorbase.org/). The genomes of Ceratitis capitata [44] and Musca domestica [45] were downloaded from NCBI database. We obtained the genome data of Apis mellifera, Bombyx mori, Tribolium castaneum, Acyrthosiphon pisum from Hymenoptera Genome database (http:// hymenopteragenome.org/), Silkworm Genome database 
(http://silkworm.genomics.org.cn/), Beetlebase (http:// www.beetlebase.org/) and AphidBase (http://bipaa.genouest.org/is/aphidbase/) respectively. Firstly, candidate B. dorsalis TRP genes were identified by TBLASTN searches against genome and transcriptomes with an E-value cutoff of $1 \mathrm{e}^{-5}$, using known TRP protein sequences of D. melanogaster, Apis mellifera and humans (Homo sapiens). Then, candidate genes were further verified using BLASTP versus non-redundant NCBI protein sequences without species limits and with a cut-off e-value of $1 \mathrm{e}^{-5}$ [46]. The same procedure was used to identify TRP genes of other Diptera species and Brv genes of A. mellifera, B. mori, T. castaneum, $A$. pisum by a homology-based approach.

\section{Reverse transcription PCR (RT-PCR)}

To investigate the splice variants of TRP channels and to confirm the identified Pkd2 channels in B. dorsalis, primers (Additional file 1: Table S1) were designed to amplify part of the open reading frames (ORFs) of TRPL, $P y x$ and $P k d 2$ genes. Total RNA was isolated from the compound eyes, abdomens, and whole bodies of six adult flies using the Trizol reagent (Invitrogen, Carlsbad, CA, USA) and was treated with RQ1 DNase I (Promega, Madison, WI) to eliminate genomic DNA (gDNA). Singlestrand complementary DNA (cDNA), synthesized from the Total RNA (1 $\mu \mathrm{g})$ using a RevertAid First Strand cDNA Synthesis Kit (Thermo Scientific), was then used as a template for PCRs. All amplifications were carried out using Phusion high-fidelity DNA polymerase (Thermo Scientific) according to the manufacturer's instructions and products were separated to check their sizes via electrophoresis onto a $1.0 \%$ agarose gel. Purified PCR products were then cloned into a pEASY-Blunt Zero Cloning Vector (TransGen, Beijing, China) following the manufacturer's instructions before being sequenced.

\section{Phylogenetic analysis and sequence alignment}

In order to classify the TRP channels we identified into different subfamilies, amino acid sequences were phylogenetically characterized in each case. Thus, protein structural information for all candidate TRPs was identified via an InterProScan (http://www.ebi.ac.uk/ Tools/InterProScan/) search, and sequences were aligned using the software ClustalW2 (http://www.ebi.ac.uk/Tools/msa/clustalw2/). Phylogenetic trees were constructed using MEGA 5.05 with 1000 bootstrap replicates based on the Maximum Likelihood method. Poisson correction model and the partial deletion method for gaps were used. Branch support values were expressed as percentages. The accession numbers of all the TRP channels used in this study are listed in Additional file 1: Table S2.

\section{Quantitative real time PCR (qRT-PCR)}

Samples from different developmental stages were collected to investigate the spatiotemporal distribution of TRPs in B. dorsalis; these included eggs (between one hour and two hours old), larvae (one day, four days, and seven days old), pupae (one day, four days, and seven days old), immature males and females (one day old), and mature males and females (13 days old). 13-day- old adults mixed with the same number of males and females were dissected into antennae, compound eyes, mouthparts, legs, wings, the brain, fat bodies, gut, Malpighian tubules, ovaries and testes. For temporal distribution analysis, approxmatily 100 eggs were used for a pool and 6 were used for a pool for the rest stages. For tissue distribution, 30 adults were included in a pool. The replicates are different pools of individuals from independent cages on the same day. At least three sample biological replicates were carried out in each case.

We extracted RNA using the TRIzol reagent (as discussed above) and measured RNA quantities using a Nanodrop 2000 spectrophotometer (Thermo Scientific Inc., Bremen, Germany). Reverse transcription was then performed with $1 \mu \mathrm{g}$ of RNA using TransScript one-step gDNA removal and cDNA Synthesis SuperMix (TransGen Biotech, China). Synthesized cDNA was then used as a template for qRT-PCR; this was performed with a Stratagene Mx3000P thermal cycler (Agilent Technologies, Wilmington, DE). The reaction mixtures used in each case contained $12.5 \mu \mathrm{L}$ of $2 \times$ TransStart Top Green qPCR SuperMix (TransGen Biotech, Beijing, China), $0.4 \mu \mathrm{L}$ of positive reference dye, $0.4 \mu \mathrm{L}$ of each primer $(0.2 \mu \mathrm{M})$, and $2 \mu \mathrm{L}$ of template cDNA. Sterile distilled water was then added to these mixtures up to a final volume of $25 \mu \mathrm{L}$. The thermal cycling conditions used in this study comprised $30 \mathrm{~s}$ at $95{ }^{\circ} \mathrm{C}, 40$ cycles at $95{ }^{\circ} \mathrm{C}$ for five seconds each, and $34 \mathrm{~s}$ at $60{ }^{\circ} \mathrm{C}$. Three sample replicates were performed for each group, and no-template negative controls were included in each run to detect possible contamination or carryover. A series of gene-specific primers were designed for qRT-PCR using the software Primer 3 (http://bioinfo.ut.ee/primer3-0.4.0/) (Additional file 1: Table S1); these primers were utilized to investigate the relative expression of selected samples, while a melting curve analysis was performed between $60{ }^{\circ} \mathrm{C}$ to $95{ }^{\circ} \mathrm{C}$ for all reactions to ensure the specificity and consistency of generated products. The specificity of all qRT-PCR reaction products was established via electrophoresis on a $1.0 \%$ agarose gel prior to sequencing, and all experiments were performed independently at least twice to ensure their reliability and reproducibility.

We quantified the transcript levels of different genes using the $2^{-\triangle \Delta C T}$ method [47], and ensured comparable quantities of cDNA by amplifying $\alpha$-tubulin as a 
reference gene as this possesses excellent spatiotemporal expression stability in $B$. dorsalis [48]. We set the lowest expression level stage to one for this analysis in order to calibrate relative levels in different development stages; relative expression levels were therefore assessed by comparing the situation in each target gene in other developmental stages to that of the lowest stage. The same developmental stage method was also applied for analysis of relative expression levels in various tissues. The data of relative expression levels in different development stages and various tissues were analyzed using one-way analysis of variance (ANOVA), followed by a Tukey's multiple comparison test when significant differences were tested. For the comparison of expression differences between splice forms, unpaired $t$ test were applied. All statistical analyses were performed using the software GraphPad Prism 5.0 (San Diego, CA).

\section{Additional file}

Additional file 1: Table S1. Primers used in this study. Table S2. Accession number of TRP channels used in this study. (DOCX $28 \mathrm{~kb}$ )

\section{Acknowledgements}

The authors sincerely thank Yu-peng Chen for assistance in feeding the oriental fruit fly. The authors also thank the editor and reviewers for their comments, which are valuable and very helpful for revising and improving our paper.

\section{Availability of data and material}

All sequence data that support the findings of this study were listed in Additional file 1: Table S2.

\section{Funding}

This research was supported by National key research and development project (No. 2016YFC1201200) and Natural Science Foundation of Guangdong Province (2018A030310204).

\section{Authors' contributions}

YXQ and YYL conceived and designed the experimental plan. HAS, XB and TZ preformed the experiments. YXQ and HAS analyzed and interpreted the experimental data. YYL, YXQ, and HAS drafted the manuscript. All authors read and approved the final manuscript.

\section{Ethics approval and consent to participate}

The material we used in this study is a notorious agricultural pest, the oriental fruit fly Bactrocera dorsalis. The strain in this study is a wide used laboratory strain for the oriental fruit fly functional researches. Therefore, it does not involve ethical issues and not need relevant permission.

\section{Consent for publication}

Not applicable.

\section{Competing interests}

The authors declare that they have no competing interests.

\section{Publisher's Note}

Springer Nature remains neutral with regard to jurisdictional claims in published maps and institutional affiliations.
Received: 17 July 2018 Accepted: 31 August 2018

Published online: 14 September 2018

\section{References}

1. Venkatachalam K. Montell C. TRP channels. Annu Rev Biochem. 2007; 76:387-417

2. Fowler MA, Montell C. Drosophila TRP channels and animal behavior. Life Sci. 2013;92(8):394-403.

3. Montell C. Drosophila TRP channels. Pflügers Arch. 2005;451:19-28.

4. Zermoglio PF, Latorre-Estivalis JM, Crespo JE, Lorenzo MG, Lazzari CR. Thermosensation and the TRPV channel in Rhodnius prolixus. J Insect Physiol. 2015;81:145-56.

5. Chouquet B, Debernard S, Bozzolan F, Solvar M, Maïbèche-Coisné M. Lucas P. A TRP channel is expressed in Spodoptera littoralis antennae and is potentially involved in insect olfactory transduction. Insect $\mathrm{Mol}$ Biol. 2009;18:213-22.

6. Montell C, Rubin GM. Molecular characterization of the Drosophila trp locus: a putative integral membrane protein required for phototransduction. Neuron. 1989;2:1313-23.

7. Andrew B. Thermotaxis, circadian rhythms, and TRP channels in Drosophila. Temperature. 2015;2:227-43.

8. Zhang $M$, Li X, Zheng $H$, Wen $X$, Chen S, Ye J, Tang S, Yao F, Li Y, Yan Z. Brv1 is required for Drosophila larvae to sense gentle touch. Cell Rep. 2018;23:23.

9. Peng $G$, Shi X, Kadowaki T. Evolution of TRP channels inferred by their classification in diverse animal species. Mol Phylogenet Evol. 2015;84:145-57.

10. Matsuura H, Sokabe T, Kohno K, Tominaga M, Kadowaki T. Evolutionary conservation and changes in insect TRP channels. BMC Evol Biol. 2009;9:228.

11. Christenson L, Foote RH. Biology of fruit flies. Annu Rev Entomol. 1960;5: 171-92.

12. Clarke AR, Armstrong KF, Carmichael AE, Milne JR, Raghu S, Roderick GK, Yeates DK. Invasive phytophagous pests arising through a recent tropical evolutionary radiation: the Bactrocera dorsalis complex of fruit flies. Annu Rev Entomol. 2005:50:293-319.

13. Hsu JC, Feng HT, Resistance WWJ. Synergistic effects of insecticides in Bactrocera dorsalis (Diptera: Tephritidae) in Taiwan. J Econ Entomol. 2004;97: 1682-8.

14. Huang Y, Lu XP, Wang LL, Wei D, Feng ZJ, Zhang Q, Xiao LF, Dou W, Wang JJ. Functional characterization of NADPH-cytochrome $\mathrm{P} 450$ reductase from Bactrocera dorsalis: possible involvement in susceptibility to malathion. Sci Rep. 2015;5:18394

15. Nesterov A, Spalthoff $C$, Kandasamy $R$, Katana R, Rankl NB, Andrés $M$, Jähde $P$, Dorsch JA, Stam LF. Braun FJ. TRP channels in insect stretch receptors as insecticide targets. Neuron. 2015;86:665-71.

16. Kandasamy R, London D, Stam L, von Deyn W, Zhao X, Salgado VL, Afidopyropen NA. New and potent modulator of insect transient receptor potential channels. Insect Biochem Mol Biol. 2017;84:32-9.

17. Kwon Y, Kim SH, Ronderos DS, Lee Y, Akitake B, Woodward OM, Guggino WB, Smith DP, Montell C. Drosophila TRPA1 channel is required to avoid the naturally occurring insect repellent citronellal. Curr Biol 2010; 20:1672-1678.

18. Salgado VL. Insect TRP channels as targets for insecticides and repellents. J Pestici Sci. 2017;42:1-6.

19. Kim SH. Insect GPCRs and TRP channels: putative targets for insect repellents. Interdisciplinary Bio Central. 2013;5:6.

20. Palmer CP, Aydar E. Djamgoz MB. A microbial TRP-like polycystic-kidneydisease-related ion channel gene. Biochem J. 2005;387:211-9.

21. Matsuura $H$, Sokabe $T$, Kohno $K$, Tominaga $M$, Kadowaki T. From worm to man: three subfamilies of TRP channels. BMC Evol Biol 2009; 9:228.

22. Turner H, Armengol K, Patel A, Himmel N, Sullivan L, lyer SC, Bhattacharya S, lyer EP, Landry C, Galko M. The TRP channels Pkd2, NompC, and Trpm act in cold-sensing neurons to mediate unique aversive behaviors to noxious cold in Drosophila. Curr Biol. 2016;26:3116.

23. Kohno K, Sokabe T, Tominaga M, Kadowaki T. Honey bee thermal/chemical sensor, AmHsTRPA, reveals neofunctionalization and loss of transient receptor potential channel genes. J Neurosci. 2010;30:12219-29.

24. Georgiev P, Okkenhaug $H$, Drews A, Wright D, Lambert S, Flick M, Carta V, Martel C, Oberwinkler J, Raghu P. TRPM channels mediate zinc homeostasis and cellular growth during Drosophila larval development. Cell Metab. 2010; 12:386-97. 
25. Hofmann T, Chubanov V, Chen X, Dietz AS, Gudermann T, Montell C. Drosophila TRPM channel is essential for the control of extracellular magnesium levels. PLoS One. 2010;5:e10519.

26. Komiya Y, Runnels LW. TRPM channels and magnesium in early embryonic development. Int J Dev Biol. 2015;59:281.

27. Hardie R, Reuss $H$, Lansdell $S$, Millar N. Functional equivalence of native lightsensitive channels in the Drosophila trp301 mutant and TRPL cation channels expressed in a stably transfected Drosophila cell line. Cell Calcium. 1997;21:431-40,

28. X-ZS X, Chien F, Butler A, Salkoff L, Montell C. TRPY, a Drosophila TRPrelated subunit, forms a regulated cation channel with TRPL. Neuron. 2000; 26:647-57.

29. Cheng $L E$, Song $W$, Looger $L L$, Jan $L Y$, Jan $Y N$. The role of the TRP channel NompC in Drosophila larval and adult locomotion. Neuron. 2010;67:373-80.

30. Akitake B, Ren Q, Boiko N, Ni J, Sokabe T, Stockand JD, Eaton BA, Montell C. Coordination and fine motor control depend on Drosophila TRPy. Nat Commun. 2015;6:7288.

31. Wicher D, Schönherr R, Heinemann SH, Derst C. TRPY channels are inhibited by CAMP and contribute to pacemaking in neurosecretory insect neurons. J Biol Chem. 2006;281:3227-36.

32. Wei J, Fu T, Yang T, Liu Y, Wang G. A TRPA1 channel that senses thermal stimulus and irritating chemicals in Helicoverpa armigera. Insect Mol Biol. 2015;24:412-21.

33. Fu T, Hull J, Yang T, Wang G. Identification and functional characterization of four transient receptor potential ankyrin 1 variants in Apolygus lucorum (Meyer-Dür). Insect Mol Biol. 2016;25:370-84.

34. Wang G, Qiu YT, Lu T, Kwon HW, Jason Pitts R, Van Loon JJ, Takken W, Zwiebel $\sqcup$. Anopheles gambiae TRPA1 is a heat-activated channel expressed in thermosensitive sensilla of female antennae. Eur J Neurosci. 2009;30:967-74.

35. Du EJ, Ahn TJ, Kwon I, Lee JH, Park J-H, Park SH, Kang TM, Cho H, Kim TJ, Kim H-W. TrpA1 regulates defecation of food-borne pathogens under the control of the duox pathway. PLoS Genet. 2016;12:e1005773.

36. Xu C, Luo J, He L, Montell C, Perrimon N. Oxidative stress induces stem cell proliferation via TRPA1/RyR-mediated $\mathrm{Ca}^{2+}$ signaling in the Drosophila midgut. eLife. 2017;6

37. Liu L, Li Y, Wang R, Yin C, Dong Q, Hing H, Kim C, Welsh MJ. Drosophila hygrosensation requires the TRP channels water witch and nanchung. Nature. 2007:450:294.

38. Lee Y, Lee Y, Lee J, Bang S, Hyun S, Kang J, Hong S-T, Bae E, Kaang B-K, Kim J. Pyrexia is a new thermal transient receptor potential channel endowing tolerance to high temperatures in Drosophila melanogaster. Nat Genet. 2005;37:305.

39. Gallio M, Ofstad TA, Macpherson LJ, Wang JW, Zuker CS. The coding of temperature in the Drosophila brain. Cell. 2011;144:614.

40. Gao Z, Ruden DM, Lu X. PKD2 cation channel is required for directional sperm movement and male fertility. Curr Biol. 2003;13:2175-8.

41. Köttgen M, Hofherr A, Li W, Chu K, Cook S, Montell C, Watnick T. Drosophila sperm swim backwards in the female reproductive tract and are activated via TRPP2 ion channels. PLoS One. 2011;6:e20031.

42. Chang $\mathrm{CL}$, Vargas Rl, Caceres C, Jang E, Cho IK. Development and assessment of a liquid larval diet for Bactrocera dorsalis (Diptera: Tephritidae). Ann Entomol Soc Am. 2006;99:1191-8.

43. Liu H, Zhao XF, Fu L, Han YY, Chen J, Lu YY. BdorOBP2 plays an indispensable role in the perception of methyl eugenol by mature males of Bactrocera dorsalis (Hendel). Sci Rep. 2017;7:15894.

44. Papanicolaou A, Schetelig MF, Arensburger P, Atkinson PW, Benoit JB, Bourtzis K, Castañera P, Cavanaugh JP, Chao H, Childers C. The whole genome sequence of the Mediterranean fruit fly, Ceratitis capitata (Wiedemann), reveals insights into the biology and adaptive evolution of a highly invasive pest species. Genome Biol. 2016;17:192.

45. Scott JG, Warren WC, Beukeboom LW, Bopp D, Clark AG, Giers SD, Hediger M, Jones AK, Kasai S, Leichter CA. Genome of the house fly, Musca domestica L., a global vector of diseases with adaptations to a septic environment. Genome Biol. 2014;15:466

46. Liu NY, Xu W, Dong SL, Zhu JY, Xu YX, Anderson A. Genome-wide analysis of ionotropic receptor gene repertoire in Lepidoptera with an emphasis on its functions of Helicoverpa armigera. Insect Biochem Mol Biol. 2018;99:37-53.

47. Livak KJ, Schmittgen TD. Analysis of relative gene expression data using real-time quantitative PCR and the 2(-Delta Delta C(T)) method. Methods. 2001;25:402-8

48. Shen GM, Jiang HB, Wang XN, Wang JJ. Evaluation of endogenous references for gene expression profiling in different tissues of the oriental fruit fly Bactrocera dorsalis (Diptera: Tephritidae). BMC Mol Biol. 2010;11:1.

Ready to submit your research? Choose BMC and benefit from:

- fast, convenient online submission

- thorough peer review by experienced researchers in your field

- rapid publication on acceptance

- support for research data, including large and complex data types

- gold Open Access which fosters wider collaboration and increased citations

- maximum visibility for your research: over $100 \mathrm{M}$ website views per year

At BMC, research is always in progress.

Learn more biomedcentral.com/submissions 